\title{
Negative-pressure wound therapy for management of chronic neuropathic noninfected diabetic foot ulcerations - short-term efficacy and long-term outcomes
}

\author{
S Borys ${ }^{1,2} \cdot \mathrm{J} \mathrm{Hohendorff}^{1,2} \cdot$ T Koblik $^{1} \cdot$ P Witek $^{1,2} \cdot$ AH Ludwig-Slomczynska $^{3} \cdot$ C Frankfurter $^{4} \cdot$ B Kiec-Wilk $^{1,2}$. \\ MT Malecki ${ }^{1,2}$
}

Received: 4 June 2018 / Accepted: 30 July 2018 / Published online: 11 August 2018

(c) The Author(s) 2018

\begin{abstract}
Purpose Negative pressure wound therapy (NPWT) is an adjunct method used in the treatment of diabetic foot ulceration (DFU). Real world data on its effectiveness and safety is scarce. In this prospective observational study, we assessed the short-term efficacy, safety, and long-term outcomes of NPWT in patients with type 2 diabetes (T2DM) and neuropathic, noninfected DFUs.

Methods Based on wound characteristics, mainly area ( $>1$ vs. $\left.\leq 1 \mathrm{~cm}^{2}\right), 75$ patients with DFUs treated in an outpatient clinic were assigned to NPWT $(n=53)$ or standard therapy $(n=22)$. Wound area reduction was evaluated after $8 \pm 1$ days. Longterm outcomes assessed included complete ulceration closure and recurrence rate.

Results Patients assigned to NPWT were characterized by greater wound area $\left(15.7\right.$ vs. $\left.2.9 \mathrm{~cm}^{2}\right)$. Reduction in wound area was found in both the NPWT $\left(-1.1 \mathrm{~cm}^{2},-10.2 \%, p=0.0001\right)$ and comparator group $\left(-0.3 \mathrm{~cm}^{2},-18.0 \%, p=0.0038\right)$. No serious adverse events related to NPWT were noted. Within 1 year, 55.1\% (27/49) of DFUs were closed in the NPWT group and $73.7 \%(14 / 19)$ in the comparator group $(p=0.15)$. In the logistic regression, wound duration and smaller initial area, but not treatment mode, were associated with closure. One-year follow-up after DFU resolution revealed an $\sim 30.0 \%$ recurrence rate in both groups $(p=0.88)$.

Conclusions NPWT is a safe treatment for neuropathic, nonischemic, and noninfected DFU in patients with T2DM, although this observational study did not prove its effectiveness over standard therapy. Additionally, we report a high rate of both closure and recurrence of ulcers, the latter irrespective of initial ulcer area.
\end{abstract}

Keywords Negative-pressure wound therapy $•$ Diabetic foot syndrome $\cdot$ Type 2 diabetes

These authors contributed equally: Borys S, Hohendorff $\mathrm{J}$

MT Malecki

malecki_malecki@yahoo.com

maciej.malecki@uj.edu.pl

1 Department of Metabolic Diseases, University Hospital in Krakow, Krakow, Poland

2 Department of Metabolic Diseases, Jagiellonian University Medical College, Krakow, Poland

3 Center for Medical Genomics OMICRON, Jagiellonian University Medical College, Krakow, Poland

4 Faculty of Medicine, University of Toronto, Toronto, Canada

\section{Introduction}

Life expectancy in people with diabetes mellitus, particularly the type 2 form of the disease, is shorter compared to the general population [1]. This is mainly attributable to its chronic complications, such as coronary artery disease, stroke, and renal failure. Diabetic foot syndrome (DFS), frequently occurring together with ulceration, is another prominent complication. The pathomechanism of DFS is complex and involves diabetic neuropathy, ischemia, and impaired function of the immune system [2]. DFS is associated with a high rate of hospitalizations and a 20 -fold increase in the risk of lower limb amputations [3, 4]. Foot ulcers precede more than $80 \%$ of nontraumatic lower extremity amputations in patients with diabetes [5, 6]. DFS is also associated with increased mortality $[7,8]$. 
In spite of new therapies for diabetes mellitus that have become available in the recent decades, DFS still affects thousands of patients worldwide and constitutes a large medical, organizational, and economic problem. There are a number of approaches in the treatment of DFS with ulceration that are used either subsequently or simultaneously, depending on the type of the wound, accessibility, and local guidelines [9]. This list includes surgical debridement of the injury bed, off-loading of the affected foot, systemic administration of wide-spectrum antibiotics when infection is present, optimization of glycemic control, and endovascular treatment (angioplasty and stenting) or surgery for peripheral artery disease if applicable. Nevertheless, in some patients, these conventional procedures are not effective, resulting in prolonged healing of foot ulcerations. Among adjuvant methods that appear to accelerate wound healing, negative-pressure wound therapy (NPWT) seems to be particularly effective in diabetic foot ulcerations. NPWT involves the use of a device that is connected to the wound bed through a special set and generates a negative pressure [10]. Proposed mechanisms of its action at the tissue and cellular level include reduction of the edema, local blood flow improvement, granulation and angiogenesis induction, epithelialization of the wound borders, and facilitation of cell migration and proliferation [10]. Macrostrain mechanisms of NPWT involve both union of wound edges and removal of exudates with infectious materials from the wound bed. NPWT has been shown to be safe and effective in wound healing, especially in postoperative lesions. Its efficacy in diabetic foot ulcers was confirmed by several inpatient randomized controlled trials [11, 12]. However, there is still a need for real world observational data from outpatient clinics concerning its use in specific ulcer subtypes.

The aims of this prospective observational study were: (1) to assess the short-term efficacy and safety data of NPWT use in patients with type 2 diabetes mellitus (T2DM) and concomitant neuropathic, nonischemic, noninfected foot wounds; (2) to collect information about long-term outcomes in these patients and compare them with patients not exposed to NPWT and patients with different wound characteristics, notably smaller ulcer areas.

\section{Subjects and methods}

The patients included in this prospective clinical observation were participants of the research project on the molecular mechanisms of NPWT [13]. The total study group consisted of 75 consecutive patients with T2DM and concomitant foot ulceration(s) treated between 2014 and 2018 in the Department of Metabolic Diseases of the University Hospital in Krakow, a tertiary academic outpatient center for patients with DFS in southern Poland. Patients were qualified to the study if they had peripheral diabetic polyneuropathy (diagnosed with at least two standard clinical tests) complicated by superficial neuropathic, noninfected, and nonischemic foot ulcerations. Exclusion criteria included active osteomyelitis and active Charcot neuroarthropathy that were verified by clinical examination (depth of wound, probe-to-bone test) and radiographs of the affected foot, as well as clinically significant ischemia defined as the lack of pulses of both main foot arteries and/or an anklebrachial index $<0.9$. Subjects were assigned to the standard therapy alone or combined with NPWT for $8 \pm 1$ days, a duration routinely used in our clinical practice. Allocation of patients with T2DM to study arms was not random but based on wound characteristics and pre-specified criteria. Patients were assigned to the NPWT group if they had neuropathic, noninfected ulcerations of an area $>1 \mathrm{~cm}^{2}$ on one foot, or to the comparator group if they had ulcerations of an area $\leq 1 \mathrm{~cm}^{2}$ or bilateral DFS. Conversely, patients were allocated to the comparator group, if they had ulcerations of an area $>1 \mathrm{~cm}^{2}$, if technical difficulties existed (e.g. unfavorable localization for NPWT application), or if the patient did not consent to NPWT. At the initial visit, patients were assigned to treatment including, or not including, the use of NPWT. The NPWT dressing was changed 3-5 days after the initial visit, and after another 3-5 days, the treatment was terminated. Patients assigned to the comparator group had control visits at the same time intervals. At each timepoint, a standard clinical examination and wound debridement was performed. We used a commercial NPWT system (Renasys EZ Plus/Smith \& Nephew, London, United Kingdom) with a portable device and adjusted fluid canister. Continuous negative pressure of $-120 \mathrm{mmHg}$ was applied during therapy. Renasys $\mathrm{F}$ foams were cut and fitted to the wound bed, and Renasys soft ports were used as above when dressings were changed. The study protocol was approved by the Jagiellonian University Bioethical Committee and was in accordance with the Declaration of Helsinki. Informed consent was obtained from all individual participants included in the study.

After this initial time period, all subjects remained under routine medical care and received standard therapy in our center. We performed a follow-up to assess long-term results of ulcer healing and potential ulcer recurrence in both study groups with different baseline ulcer characteristics. After the initial phase, all patients remained under routine outpatient care. During the whole period of treatment and after wound closure, telephone contact with the clinic center was also possible for patients. A standard offloading method through the use of appropriate footwear, two crutches and/or a wheelchair was advised during therapy. Moreover, basic rehabilitation techniques with a minimal wound damage risk profile were advised. For 
homecare, patients were also advised to change a standard wound dressings two times a day after typical basic hygiene procedures using sterile gauze and saline and/or colorless antiseptic fluids. During both the initial and follow-up phases, ulcer area was measured at every visit using the MOWA Mobile Wound Analyzer application (Healthpath, Italy).

For the initial phase, we defined the main outcome as a change in wound area within each study group expressed as an absolute value, as well as a percentage. The list of outcomes for the follow-up phase included closure and recurrence rate in each group, as well as the comparison between groups. A full epithelialization of the wound bed without

Table 1 Baseline clinical characteristics and biochemical measurements of the study groups

\begin{tabular}{|c|c|c|c|}
\hline & NPWT group & Comparator group & $p$ value \\
\hline Number of cases; $n$ & 53 & 22 & NA \\
\hline $\mathrm{Sex} ; n \mathrm{M} / \mathrm{F}, \% \mathrm{M}$ & $45 / 8,84.9 \%$ & $17 / 5,77.3 \%$ & 0.42 \\
\hline Age; years ${ }^{\mathrm{a}}$ & $65.4 \pm 8.6$ & $64.2 \pm 6.8$ & 0.54 \\
\hline Duration of diabetes; years ${ }^{a}$ & $14.3 \pm 8.0$ & $17.1 \pm 7.4$ & 0.17 \\
\hline BMI; $\mathrm{kg} / \mathrm{m}^{2}$ a & $29.8 \pm 5.1$ & $30.5 \pm 4.9$ & 0.60 \\
\hline Insulin therapy; $n \mathrm{Y} / \mathrm{N}, \% \mathrm{Y}$ & $50 / 3,94.3 \%$ & $20 / 1,95.2 \%$ & 0,87 \\
\hline Daily dose of insulin; $\mathrm{U}^{\mathrm{a}}$ & $55.3 \pm 27.5$ & $55.0 \pm 25.9$ & 0.97 \\
\hline HbA1c; $\%^{\mathrm{a}}$ & $7.0 \pm 1.3$ & $7.4 \pm 1.5$ & 0.30 \\
\hline $\mathrm{HbA} 1 \mathrm{c}, \mathrm{mmol} / \mathrm{mol}^{\mathrm{a}}$ & $53.5 \pm 13.9$ & $57.4 \pm 16.8$ & 0.30 \\
\hline $\begin{array}{l}\text { eGFR CKD EPI; } \mathrm{ml} / \mathrm{min} / \\
1,73 \mathrm{~m}^{2} \text { a }\end{array}$ & $79.6 \pm 21.7$ & $74.5 \pm 21.1$ & 0.35 \\
\hline $\begin{array}{l}\mathrm{eGFR}<60 \mathrm{ml} / \mathrm{min} / 1,73 \mathrm{~m}^{2}, \\
\%(n)\end{array}$ & $20.8 \%$ & $31.8 \%(7)$ & 0.31 \\
\hline Total cholesterol; $\mathrm{mmol} / \mathrm{l}^{\mathrm{a}}$ & $3.3 \pm 0.8$ & $3.4 \pm 0.9$ & 0.75 \\
\hline $\mathrm{HDL} ; \mathrm{mmol} / \mathrm{l}^{\mathrm{a}}$ & $0.9 \pm 0.4$ & $0.8 \pm 0.1$ & 0.90 \\
\hline $\mathrm{LDL} ; \mathrm{mmol} / 1^{\mathrm{a}}$ & $2.0 \pm 0.8$ & $2.1 \pm 0.7$ & 0.45 \\
\hline Triglycerides; $\mathrm{mmol} / \mathrm{l}^{\mathrm{a}}$ & $1.3 \pm 0.6$ & $1.5 \pm 0.7$ & 0.60 \\
\hline $\begin{array}{l}\text { Smoking; } n \\
\text { current/former/never }\end{array}$ & $4 / 27 / 18$ & $3 / 9 / 8$ & 0.61 \\
\hline
\end{tabular}

${ }^{a}$ Data presented as mean \pm SD the presence of discharge or crust was required to consider the ulcer closed. We also followed deaths and large amputations.

Statistical analysis was performed using Statistica Software v. 13.0 (TIBICO Software, Palo Alto, CA, USA). A $p$ value of $<0.05$ was considered significant. Parametric $t$ tests or nonparametric $U$ tests were performed to describe baseline clinical characteristic of the study groups. To assess changes in wound areas after treatment within study groups, Wilcoxon tests were performed. Clinical factors affecting the closure within 12 months in the study groups were assessed with logistic regression. The list of independent variables consisted of age, sex, BMI, initial mode of treatment (NPWT vs. standard), wound duration, wound area, diabetes duration, HbA1c value, kidney function (GFR), and smoking status.

\section{Results}

Overall, we included 75 patients with T2DM in this observation. There were 53 patients assigned to the NPWT group and 22 to the comparator group. Most of patients were assigned to the comparator group based on the wound area (ulcerations $\leq 1 \mathrm{~cm}^{2}$ ), rather than other criteria. No differences between the study groups in terms of baseline characteristics were found, as summarized in Table 1. The NPWT group was characterized by greater wound areas than in the comparator group (Table 2), which was in line with how patients were allocated. Mean wound area was $15.7 \pm 14.6 \mathrm{~cm}^{2}$ in the NPWT group and $2.9 \pm 4.4 \mathrm{~cm}^{2}$ in the comparator group. However, after removal of 4 outliers with a large ulcer area, the mean wound area in comparator group was $1.6 \pm 1.2 \mathrm{~cm}^{2}$.
Table 2 Wound-related study characteristics and outcomes

\begin{tabular}{llll}
\hline & NPWT group & Comparator group & $p$ value \\
\hline Wound duration, weeks $^{\mathrm{a}}$ & $21.1 \pm 24.7$ & $14.4 \pm 14.3$ & 0.10 \\
Wound area; cm $^{2}$ a & $15.7 \pm 14.6$ & $2.9 \pm 4.4$ & 0.0000 \\
Wound area reduction after $8 \pm 1$ days; cm $^{2}$ a & $1.1 \pm 1.7$ & $0.3 \pm 0.7$ & $0.20^{\mathrm{b}}$ \\
Wound area reduction after $8 \pm 1$ days; \% & $10.2 \pm 14.41$ & $18.0 \pm 15.7$ & $0.04^{\mathrm{b}}$ \\
Complete ulcer resolution within 1 year; \% (n) & $55.1(27 / 49)$ & $73.7(14 / 19)$ & 0.15 \\
Ulcer recurrences within 1 year after complete & $29.2(7 / 24)$ & $31.3(5 / 16)$ & 0.88 \\
ulcer resolution; \% $(n)$ & & & \\
Median follow-up, months, mean SD, median, IQ & $21.1 \pm 9.1$ & $23.8 \pm 10.4$ & 0.26 \\
range & $21.1(\mathrm{IQR}:$ & $29.8(\mathrm{IQR}: 15.2-31.7)$ & \\
& $15.9-28.3)$ & & 0.11 \\
Large amputations; $n$ & 0 & 1 & 0.61 \\
Deaths; $n$ & 3 & 2 & \\
\hline
\end{tabular}

${ }^{\mathrm{a}}$ Data presented as mean $\pm \mathrm{SD}$

${ }^{\mathrm{b}}$ The $p$-value for the reduction in wound area after $8 \pm 1$ days in the NPWT was $p=0.0001$, while in the comparator group it was 0.0038 


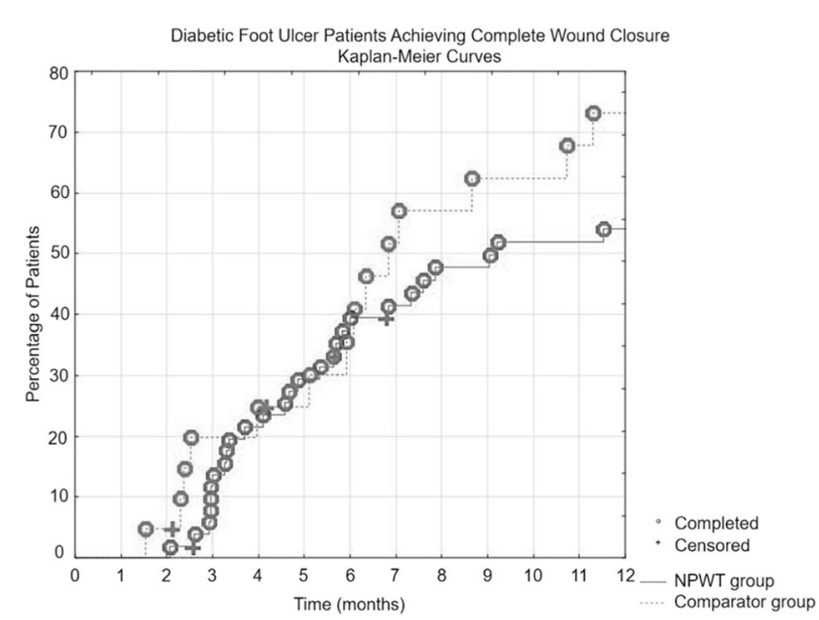

Fig. 1 Kaplan-Meier survival curves showing percentage of patients with diabetic foot syndrome achieving complete wound closure

Reduction in wound area after $8 \pm 1$ days was found in both the NPWT and comparator group $\left(-1.1 \pm 1.7 \mathrm{~cm}^{2}\right.$, $-10.2 \% \pm 14.4 \%, p=0.0001$ and $-0.3 \pm 0.6,-18.0 \% \pm$ $15.7 \%, p=0.0038$, respectively). During the initial phase, we did not note either local or systemic serious complications with NPWT, including pain, cellulitis, infections, necrosis, hemorrhage, and thrombosis. There were 2 patients from the NPWT group and none from comparator group that were lost to follow-up at the end of the initial phase of the study.

After the initial phase, all patients were observed on average every $5.2 \pm 2.6$ weeks until the whole ulceration was closed. Within 1 year, $55.1 \%$ (27/49) of foot ulcerations were healed in the NPWT group and 73.7\% (14/19) in the comparator group $(p=0.1525)$. During the median follow-up (time to complete ulceration closure or time to loss of follow-up) of 7.6 months (IQR: 4.1-17.3 months) in NPWT group and 6.3 months (IQR: 4.0-11.3 months), a complete ulcer closure was achieved in $78.4 \%$ (40/51) and $77.3 \%$ (17/22) patients, respectively $(p=0.9126)$. Based on the logistic regression, the factors associated with complete wound closure within one year among the pooled group were shorter wound duration (OR: 0.885, 95\% CI: $0.799-0.982)$ and smaller wound area (OR: $0.905,95 \%$ CI: 0.830-0.986), but not other factors, including mode of treatment. Kaplan-Meier survival curves are shown in Fig. 1. One-year follow-up after ulceration resolution revealed $29.1 \%(7 / 24)$ and $31.3 \%(5 / 16)$ of recurrences in foot ulceration in the NPWT and comparator group, respectively $(p=0.8880)$. During the median entire follow-up of 21.8 months (IQR: 15.6-29.9), a large amputation occurred in one patient and 5 patients died ( 3 of them after complete wound healing; 3 deaths occurred in patients from the NPWT group and 2 from the comparator group). Moreover, there were 5 patients with unhealed wounds lost to followup (4 patients from the NPWT group after 3, 7, 15, and 16 months and 1 patient from comparator group after 4 months). Additionally, 8 patients were lost to follow-up after complete ulcer healing (mean $4.3 \pm 4.8$ months) Treatment outcomes are summarized in Table 2.

\section{Discussion}

We present the results of a prospective clinical outpatient observation of patients with T2DM and concomitant foot ulcers. We report the effective initial, rapid response to NPWT in patients with neuropathic, nonischaemic, and noninfected foot wounds. Additionally, long-term follow-up data with a rate of both healing and recurrence are provided for ulcers of different areas.

Patients with neuropathic, nonischemic, and noninfected plantar ulcerations constitute approximately one-quarter of patients in diabetic foot clinics [14]. While closure is reached within 1-2 months in a number of affected individuals, the remaining patients experience prolonged healing and constitute a real challenge in clinical practice. The International Working Group on the Diabetic Foot (IWGDF) suggests using a nonremovable total contact cast (TTC) that has shown to be of benefit in wound healing for diabetic ulcerations with such characteristics [15-17]. However, due to its time-consuming nature and infrequent possibility for reimbursement, amongst other causes, TCC is applied only in $6 \%$ of patients who are potentially good candidates for such a treatment [18]. NPWT is another option recommended for the treatment of diabetic foot ulcers by some international expert groups [19]. Its efficacy was confirmed in 2 long-term randomized trials, both of them lasting 112 days, where it showed superiority over standard approaches or advanced moist wound therapy in the treatment of diabetic foot ulcers, reaching closure rates of 43 and $56 \%$ in the two trials, respectively [11, 12]. Evidence pertaining its efficacy in the real world has nonetheless been scarce.

In our study, a reduction of approximately $10 \%$ of the wound area was reached after 8 days of exposure to NPWT. This was similar to a $12 \%$ decrease reached in a similar study with a duration twice the length of ours [20]. It should be noted that due to the observational nature of this study, we were not able to fully objectively compare the effectiveness of NPWT over standard therapy. Unlike the Israeli study, no serious adverse events were recorded in this trial.

In our study, 55\% of ulcers in the NPWT group and over $70 \%$ in comparator group were completely healed within one year. Data analyses from 14 centers in Europe 
performed within the EURODIALE study showed a similar ulcer resolution rate [21]. The list of independent clinical predictors of complete ulcer healing includes time since the beginning of the ulceration and the magnitude of its area. Of note, the $\mathrm{HbA} 1 \mathrm{c}$ level did not influence this outcome, in line with a recently published observation [22]. Furthermore, it is well-known that end-stage renal failure and dialysis are associated with poor wound healing and limb amputation. There are also studies showing impaired wound healing in subjects with moderately reduced eGFR [23]. In our study, patients with eGFR $<60 \mathrm{ml} / \mathrm{min} / 1,73 \mathrm{~m}^{2}$ accounted for $24 \%$ of study group and eGFR was not found to be associated with healing failure. One-year follow-up revealed an ulcer recurrence rate of over $30 \%$, a finding in line with other studies [24]. Interestingly, the rate of ulcer recurrence was almost the same in both groups, in spite of a large difference in the magnitude of their initial area. This finding should alert clinicians to the fact that a high rate of recurrence is present also in ulcers of relatively small area. It has been in fact previously proposed that clinicians use the terms "ulcer remission" rather than "ulcer healing" in clinical practice [24, 25]. In the presented study, following ulcer closure, all patients received standard advice on foot ulcer recurrence prevention (e.g. maintaining adequate glycemic control, using appropriate footwear, and inspecting the foot each day). However, there is no certainty that even targeted, structured educational programs are associated with clinical benefits [26]. A population-based study revealed over a 2fold increase in the mortality risk among individuals with a history of foot ulcers [27]. The mortality risk among patients with lower limb amputation is comparable to those with malignant diseases [8]. Due to the poor prognosis associated with diabetic foot ulcers and lower limb amputations, a greater emphasis on primary and secondary prevention is needed within healthcare systems.

There are several shortcomings related to this study. First of all, the study is limited by its nonrandom, observational nature. Subsequently, there were substantial differences in the magnitude of ulcer areas in both study groups. Additionally, use of more reliable methods to exclude a substantial ischemic component of an ulcer, such as transcutaneous oxygen pressure measurement or toe systolic pressure measurement, was not available for this study. Many known and likely unknown risk factors were not included in the regression analysis. Moreover, $20 \%$ of patients were lost to follow-up, as some of them likely decided to return to their local outpatient clinics, particularly after they experienced closure of their ulcerations. However, we cannot exclude the possibility that some of them had ulcer recurrences, underwent amputations, or died. Despite these limitations, our study exhibited important real-world data on treatment of diabetic plantar ulcerations.

\section{Conclusions}

In summary, NPWT is safe for the treatment of neuropathic, nonischemic, and noninfected plantar ulcerations in patients with T2DM although this observational study did not prove its better effectiveness over standard therapy. Additionally, we report a high rate of both closure and recurrence of ulcers, the latter irrespective of initial ulcer area.

Funding The study was funded by the National Science Centre in Poland through the Opus Grant "Assessment of molecular mechanisms of negative pressure wound therapy in the treatment of neuropathic ulceration in diabetic foot syndrome" to MTM (Nr 2013/11/B/NZ5/ 03298). The Omicron laboratory (where the microarray experiments were performed) was funded by the European Union (7th Framework Programme, Call number FP7-REGPOT-2011-1, Grant number 286038).

Author contributions S.B., J.H., A.L.S., B.K.W., and M.T.M. performed the research. J.H., S.B., and C.F. analyzed the data. B.K.W., S. B., J.H., and M.T.M. designed the research study and interpreted the data. S.B., J.H., and M.T.M. wrote the paper. All authors contributed to the critical revision of the manuscript and approved its publication. M.T.M. and B.K.W. are the guarantors of this work.

\section{Compliance with ethical standards}

Conflict of interest The authors declare that they have no conflict of interest.

Ethical approval All procedures performed in studies involving human participants were in accordance with the ethical standards of the Jagiellonian University Bioethical Committee and with the 1964 Helsinki declaration and its later amendments or comparable ethical standards.

Informed consent Informed consent was obtained from all individual participants included in the study.

Open Access This article is distributed under the terms of the Creative Commons Attribution 4.0 International License (http://crea tivecommons.org/licenses/by/4.0/), which permits use, duplication, adaptation, distribution, and reproduction in any medium or format, as long as you give appropriate credit to the original author(s) and the source, provide a link to the Creative Commons license, and indicate if changes were made.

\section{References}

1. A.K. Wright, E. Kontopantelis, R. Emsley et al. Life expectancy and cause-specific mortality in type 2 diabetes: a population-based cohort study quantifying relationships in ethnic subgroups. Diabetes Care 40(3), 338-345 (2017)

2. A.J. Boulton, The diabetic foot: grand overview, epidemiology and pathogenesis. Diabetes Metab. Res. Rev. 24(Suppl 1), 3-6 (2008)

3. G.H. Skrepnek, J.L. Mills Sr., L.A. Lavery, D.G. Armstrong, Health care service and outcomes among an estimated 6.7 million ambulatory care diabetic foot cases in the U.S. Diabetes Care $\mathbf{4 0}$ (7), 936-942 (2017) 
4. N. Holman, R.J. Young, W.J. Jeffcoate, Variation in the recorded incidence of amputation of the lower limb in England. Diabetologia 55(7), 1919-1925 (2012)

5. J. Apelqvist, J. Larsson, What is the most effective way to reduce incidence of amputation in the diabetic foot? Diabetes Metab. Res. Rev. 16(Suppl 1), S75-83 (2000)

6. I.S. Muller, W.J. de Grauw, W.H. van Gerwen et al. Foot ulceration and lower limb amputation in type 2 diabetic patients in Dutch primary health care. Diabetes Care 25(3), 570-574 (2002)

7. J.W. Walsh, O.J. Hoffstad, M.O. Sullivan, D.J. Margolis, Association of diabetic foot ulcer and death in a population-based cohort from the United Kingdom. Diabet. Med. 33(11), 1493-1498 (2016)

8. M. Hoffmann, P. Kujath, A. Flemming et al. Survival of diabetes patients with major amputation is comparable to malignant disease. Diab. Vasc. Dis. Res. 12(4), 265-271 (2015)

9. N.C. Schaper, J.J. Van Netten, J. Apelqvist, B.A. Lipsky, K. Bakker, International Working Group on the Diabetic Foot (IWGDF): prevention and management of foot problems in diabetes: a Summary Guidance for Daily Practice 2015, based on the IWGDF guidance documents. Diabetes Res. Clin. Pract. 124, 84-92 (2017)

10. M.Y. Hasan, R. Teo, A. Nather, Negative-pressure wound therapy for management of diabetic foot wounds: a review of the mechanism of action, clinical applications, and recent developments. Diabet. Foot Ankle 6, 27618 (2015)

11. D.G. Armstrong, L.A. Lavery, Diabetic Foot Study Consortium.: negative pressure wound therapy after partial diabetic foot amputation: a multicentre, randomised controlled trial. Lancet 366 (9498), 1704-1710 (2005)

12. P.A. Blume, J. Walters, W. Payne, J. Ayala, J. Lantis, Comparison of negative pressure wound therapy using vacuum-assisted closure with advanced moist wound therapy in the treatment of diabetic foot ulcers. Diabetes Care 31(4), 631-636 (2008)

13. S. Borys, A. Ludwig-Galezowska, P. Kapusta et al. Negative pressure wound therapy effect in diabetic foot ulcer may be mediated through differential gene expression. Diabetologia 60 (Suppl 1), S470 (2017)

14. L. Prompers, M. Huijberts, J. Apelqvist et al. High prevalence of ischaemia, infection and serious comorbidity in patients with diabetic foot disease in Europe. Baseline results from the Eurodiale study. Diabetologia 50(1), 18-25 (2007)

15. S.A. Bus, D.G. Armstrong, R.W. van Deursen et al. IWGDF guidance on footwear and offloading interventions to prevent and heal foot ulcers in patients with diabetes. Diabetes Metab. Res. Rev. 32(Suppl 1), 25-36 (2016)

16. D.G. Armstrong, H.C. Nguyen, L.A. Lavery et al. Off-loading the diabetic foot wound: a randomized clinical trial. Diabetes Care $\mathbf{2 4}$ (6), 1019-1022 (2001)

17. M.J. Mueller, J.E. Diamond, D.R. Sinacore et al. Total contact casting in treatment of diabetic plantar ulcers. Controlled clinical trial. Diabetes Care 12(6), 384-388 (1989)

18. C.E. Fife, M.J. Carter, D. Walker, Why is it so hard to do the right thing in wound care? Wound Repair Regen. 18(2), 154-158 (2010)

19. J. Apelqvist, C. Willy, A.M. Fagerdahl et al. EWMA document: negative pressure wound therapy. J. Wound Care 26(Sup3), S1-S154 (2017)

20. E. Tamir, A.S. Finestone, I. Wiser, Y. Anekstein, G. Agar, Outpatient negative-pressure wound therapy following surgical debridement: results and complications. Adv Skin Wound Care 31, 365-369 (2018).

21. L. Prompers, N. Schaper, J. Apelqvist et al. Prediction of outcome in individuals with diabetic foot ulcers: focus on the differences between individuals with and without peripheral arterial disease. The EURODIALE Study. Diabetologia 51(5), 747-755 (2008)

22. B.K. Fesseha, C.J. Abularrage, K.F. Hines et al. Association of hemoglobin A1c and wound healing in diabetic foot ulcers. Diabetes Care 41, 1478-1485 (2018).

23. Y. He, H. Qian, L. Xu et al. Association between estimated glomerular filtration rate and outcomes in patients with diabetic foot ulcers: a 3-year follow-up study. Eur. J. Endocrinol. 177(1), 41-50 (2017)

24. D.G. Armstrong, A.J.M. Boulton, S.A. Bus, Diabetic foot ulcers and their recurrence. N. Engl. J. Med. 376, 2367-2375 (2017)

25. D.G. Armstrong, J.L. Mills, Toward a change in syntax in diabetic foot care: prevention equals remission. J. Am. Pediatr. Med. Assoc. 103(2), 161-162 (2013)

26. N.B. Lincoln, K.A. Radford, F.L. Game, W.J. Jeffcoate, Education for secondary prevention of foot ulcers in people with diabetes: a randomised controlled trial. Diabetologia 51(11), 1954-1961 (2008)

27. M.M. Iversen, G.S. Tell, T. Riise et al. History of foot ulcer increases mortality among individuals with diabetes: ten-year follow-up of the Nord-Trøndelag Health Study, Norway. Diabetes Care 32(12), 2193-2199 (2009) 